\title{
Factors Associated with the Recent Increasing Cesarean Delivery Rate at a Japanese Perinatal Center
}

\author{
Shunji Suzuki and Mariyo Nakata \\ Department of Obstetrics and Gynecology, Japanese Red Cross Katsushika Maternity Hospital, 5-11-12 Tateishi, \\ Katsushika-ku, Tokyo 124-0012, Japan \\ Correspondence should be addressed to Shunji Suzuki; czg83542@mopera.ne.jp
}

Received 29 April 2013; Accepted 8 June 2013

Academic Editors: N. A. Ginsberg, H. D. Modanlou, and K. Yang

Copyright (C) 2013 S. Suzuki and M. Nakata. This is an open access article distributed under the Creative Commons Attribution License, which permits unrestricted use, distribution, and reproduction in any medium, provided the original work is properly cited.

\begin{abstract}
Objective. We examined which specific factors contributed to the increase in Cesarean delivery rate at our hospital over a 10-year period. Methods. From January 2002 to December 2012, data on the Japanese singleton deliveries at $\geq 22$-week gestation managed at Japanese Red Cross Katsushika Maternity Hospital were collected. Potential factors associated with the increasing Cesarean delivery rate were selected according to previous studies. In this study, the incidences of intrauterine fetal demise, umbilical artery $\mathrm{pH}<7.1$, and severe perineal laceration were calculated for each year. Results. The Cesarean delivery rate at our institution increased significantly during the study period ( $17.3 \%$ in 2002 versus $23.4 \%$ in $2012, P<0.01)$. During the study period, the Cesarean delivery rates in the cases of nulliparity, preterm delivery, low birth weight $(<2,500 \mathrm{~g})$, previous Cesarean deliveries and breech presentation were increased significantly. The incidence of intrauterine fetal demise and low umbilical artery $\mathrm{pH}$ was significantly decreased, and a negative correlation was found between the Cesarean delivery rate and the incidence of low umbilical artery $\mathrm{pH}$ for each year $(r=-0.92, P<0.01)$. Conclusion. At our institute, the neonatal outcomes seemed to be improved associated with the increased Cesarean delivery rate between 2002 and 2012.
\end{abstract}

\section{Introduction}

Recently, the Cesarean delivery rate has been reported to be steadily increased in the United States [1-3]. Approximately, one-third of births in the United States are now via Cesarean delivery. The increase has been observed to be among women of all ages and race/ethnicities, in every state, and across all gestational ages. Many theories have been proffered to explain this trend, including a decrease in vaginal births after Cesarean delivery (VBAC), decreased vaginal births of breech presentation, and increased prevalence of high risk pregnancies such as advanced maternal age and some subjective indications during labor such as nonreassuring fetal status and arrest of dilation $[1,2]$. Although Cesarean delivery rates that are too low are associated with increased adverse events, Cesarean delivery rates higher than the riskadjusted expected rate for an institution have not been shown to improve maternal or neonatal outcomes, but they do add cost and unnecessary intervention [4]. Therefore, the examination of Cesarean delivery rate concerning perinatal outcomes is very important for obstetricians.

To date, however, there have not been sufficient observations concerning the Cesarean delivery rate in Japanese populations. In this study, we examined which specific factors contributed to the increase in Cesarean delivery rate at our hospital over a 10-year period.

\section{Patients and Methods}

The protocol for this study was approved by the Ethics Committee of the Japanese Red Cross Katsushika Maternity Hospital. Our hospital is one of the major perinatal centers in Tokyo, Japan (about 1,900-2,000 deliveries per year).

From January 2002 to December 2012, data on the Japanese singleton deliveries at $\geq 22$-week gestation managed at the Japanese Red Cross Katsushika Maternity Hospital were collected. Demographic information and the characteristics of labor were extracted from patient charts to 


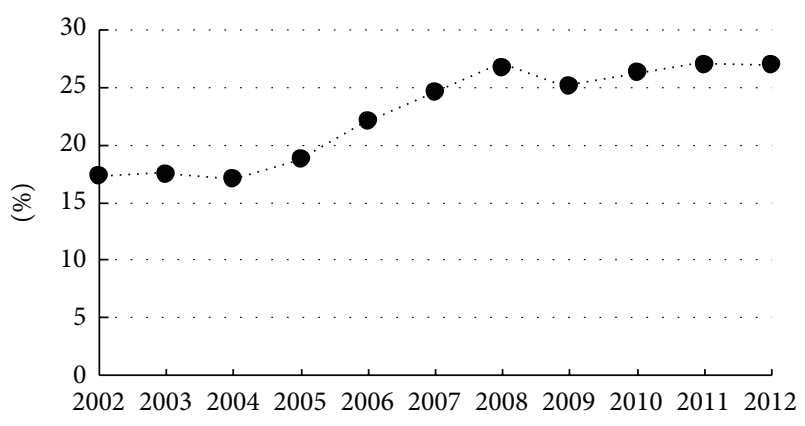

FIGURE 1: Cesarean delivery rate for each year from 2002 to 2012.

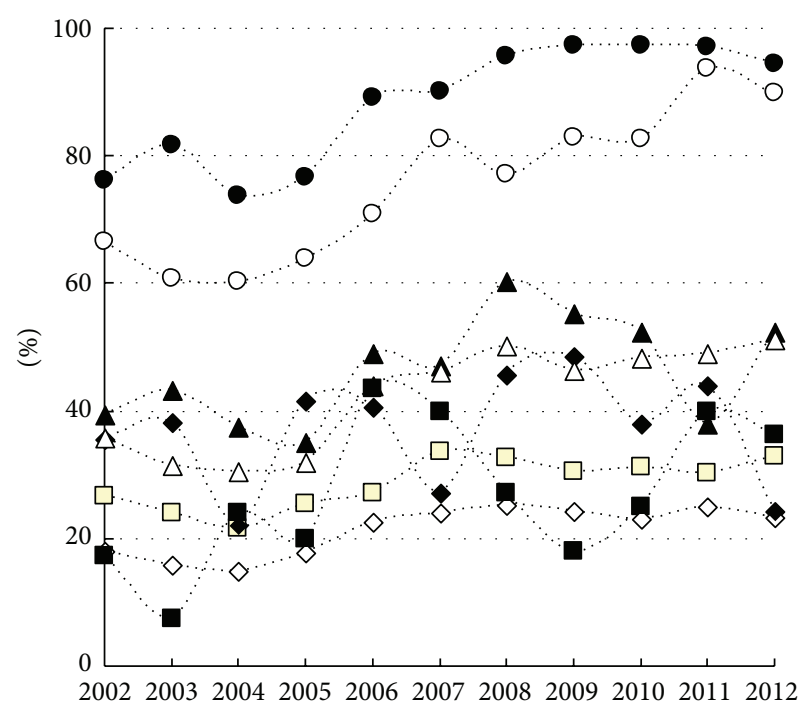

Figure 2: Changes in Cesarean delivery rate by factors $(\bullet$ breech presentation, $\circ$ previous Cesarean deliveries, $\boldsymbol{\Delta}$ preterm delivery, $\triangle$ neonatal birth weight $<2500 \mathrm{~g}$, — neonatal birth weight $\geq 4000 \mathrm{~g}$, $\square$ maternal age $\geq 35$ years, $\diamond$ pregnancy-induced hypertension, and $\diamond$ nulliparity) from 2002 to 2012.

examine the potential factors associated with the increasing Cesarean delivery rate. In this study, the factors were selected according to previous studies $[1-3,5,6]$ : nulliparity, advanced maternal age ( $\geq 35$ years), pregnancy-induced hypertension (PIH), preterm delivery, low birth weight (LBW: neonatal birth weight $<2,500 \mathrm{~g}$ ) and macrosomia (neonatal birth weight $\geq 4,000 \mathrm{~g}$ ), history of previous Cesarean deliveries, and breech presentation. PIH was defined as blood pressure $\geq 140 / 90 \mathrm{mmHg}$ measured on two or more occasions at least six hours apart with the patient at rest. In our institute, the umbilical cord $\mathrm{pH}$ was measured at all deliveries. In addition, we do not perform Cesarean sections for maternal request.

In this study, to examine the effect of the increasing Cesarean delivery rate on the obstetric outcome, the incidences of intrauterine fetal demise (IUFD), umbilical artery $\mathrm{pH}(\mathrm{UApH})<7.1$, and severe perineal laceration (perineal laceration either third- or fourth-degree laceration) were calculated for each year.

Logistic regression modeling was used to estimate Cesarean delivery rate over time and for each factor. Linear regression was performed to estimate the trend over time for each factor, Cesarean delivery rate for each factor, and obstetric outcomes. Statistical analyses were carried out using the statistical software SAS version 8.02 (SAS Institute, Cary, NC, USA), and differences with $P<0.05$ were considered significant.

\section{Results}

From January 2002 to December 2012, a total of 20,514 Japanese singleton deliveries at $\geq 22$-week gestation were managed at the Japanese Red Cross Katsushika Maternity Red Cross Hospital; 4,086 (19.9\%) of those births were delivered by Cesarean section. As shown in Figure 1, the Cesarean delivery rate at our institution increased significantly during the study period (17.3\% in 2002 versus $23.4 \%$ in 2012, $P<0.01$ ). Maternal demographic and obstetricfetal characteristics among the deliveries, which are potential factors associated with the increasing Cesarean delivery rate, during the study period are presented in Table 1 . There was a significant increase in the prevalence of advanced maternal age ( $\geq 35$ years) during the study period ( $26.6 \%$ in 2002 versus $33.0 \%$ in $2012, P<0.01)$. However, the prevalence of other factors seemed to be stable over time.

Figure 2 shows the changes in Cesarean delivery rate for each factor during the study period. As shown in Figure 2, the Cesarean delivery rates with these factors were significantly higher than the average Cesarean delivery rate among all deliveries. During the study period, the Cesarean delivery rates in the cases of nulliparity, preterm delivery, LBW, previous Cesarean deliveries, and breech presentation were increased significantly (nulliparous women: $18.0 \%$ in 2002 versus $23.4 \%$ in $2012, P<0.01$; preterm delivery: $39.5 \%$ in 2002 versus $57.5 \%$ in 2012, $P<0.01$; LBW: $35.8 \%$ in 2002 versus $57.5 \%$ in $2012, P<0.01$, previous Cesarean deliveries: $66.7 \%$ in 2002 versus $90.0 \%$ in $2012, P<0.01$; breech presentation: $76.2 \%$ in 2002 versus $94.5 \%$ in $2012, P<0.01$ ). The Cesarean delivery rates for other factors seemed to be stable over time.

Table 2 shows the changes in the incidence of IUFD, low UApH, and severe perineal laceration during the study period. During the study period, the incidence of IUFD and low UApH were significantly decreased (IUFD: $0.8 \%$ in 2002 versus $0.3 \%$ in $2012, P=0.03$; low $\mathrm{UApH}$ : $3.3 \%$ in 2002 versus $1.6 \%$ in $2012, P<0.01$ ). A negative correlation was found between the Cesarean delivery rate and the incidence of low $\mathrm{UApH}$ for each year $(r=-0.92, P<0.01)$.

\section{Discussion}

The major findings of the current study are as follows: (1) the Cesarean delivery rate at our institute increased between 2002 and 2012, (2) one of possible reasons for the increasing Cesarean delivery rate is an increase in the prevalence of advanced maternal age ( $\geq 35$ years), (3) other possible reasons for the increasing Cesarean delivery rate are the increase in Cesarean delivery rates in the cases of nulliparity, preterm delivery, LBW, previous Cesarean deliveries, and breech 


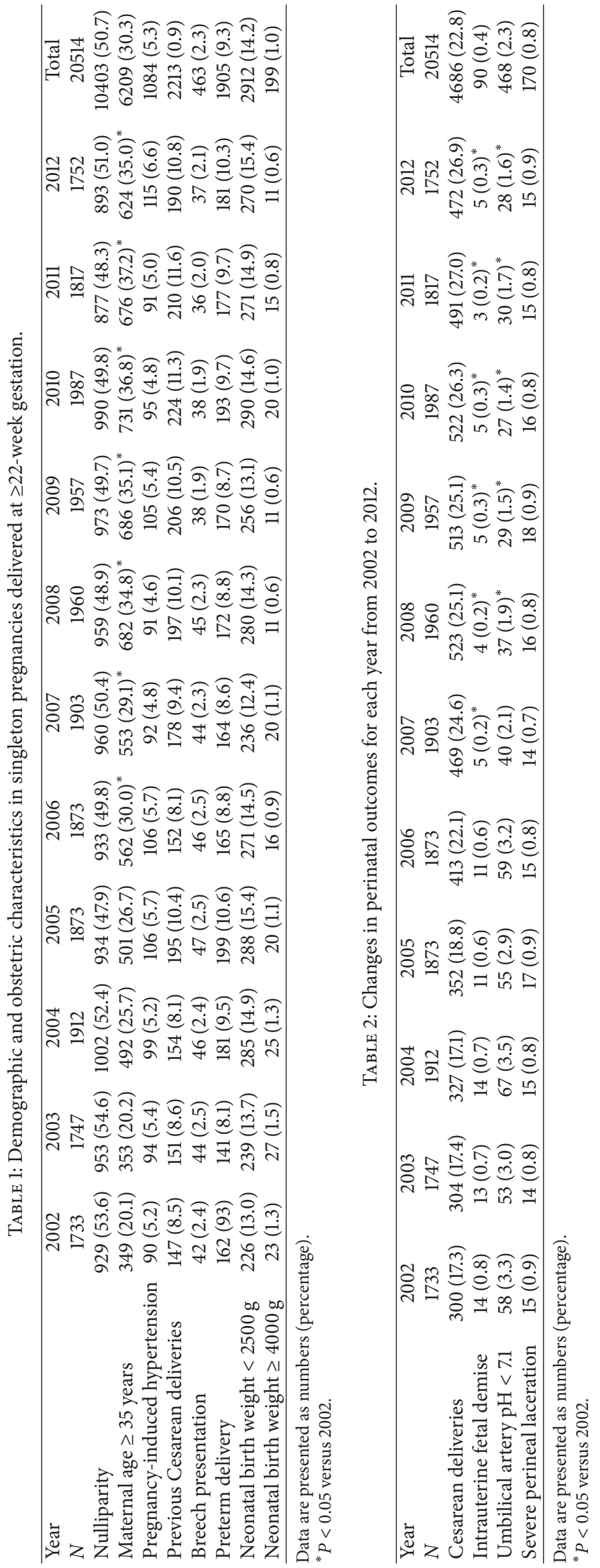


presentation, and (4) the incidence of low UApH seemed to be improved associated with the increased Cesarean delivery rate. This may be the first report examining the recent changes in Cesarean delivery rate in Japan.

We found the trends in careful correspondences for various high-risk deliveries leading to the increased Cesarean delivery rate at our institute in Japan. These trends seem to be the same as those previously reported in the USA $[1-3,5,6]$ and Japan [7-9]. For example, in a recent observation at other perinatal centers in Japan, Cesarean delivery rate was about $50 \%$ in primiparous women aged $\geq 40$ years [8].

In our institute, there has been a significant increase in the primary elective Cesarean delivery rate of singleton breech pregnancies due to medical counseling and maternal request between 2000 and 2005 [9]. This phenomenon is associated with the ACOG recommendation in 2001, which recommended Cesarean delivery for the term singleton breech [10]. An increased Cesarean delivery rate in cases with precious Cesarean deliveries seemed to be also an important factor contributing to the current results $[1-3,5]$. In our institute, intrapartum asphyxia has been observed to be independently associated with cases of trial of labor after Cesarean delivery (TOLAC) in singleton deliveries beyond 37-week gestation [11]. The asphyxia seemed to be associated with failed vacuum-extraction and/or forceps delivery during TOLAC. Therefore, our previous findings may be encouraging for the counseling of patients regarding a possible attempt at TOLAC [11]. From our impressions, these trends in delivery modes of cases with previous Cesarean deliveries and those with breech presentation have been expanded to all areas of Japan.

Unfortunately, there are some limitations in this study with the lack of taking into consideration confounders such as education/social economic status/immigrant status/antenatal cares access, all variables known to affect mode of delivery $[1-3,5,6]$. In addition, we believe that "defensive medicine" and litigation as an explicit incentive for performing Cesarean section are unlikely to play a large role. In this study, the increased Cesarean delivery rate seemed to contribute to the improvement of neonatal outcomes such as the decreased low UApH. Therefore, it appears that on a global level, the birth process is becoming more medicalised and it may need the increasing in Cesarean delivery rate in Japan. On the other hand, the increasing in Cesarean delivery rate in the past has contributed to the reduction in adverse events; however, the impact of the increasing in Cesarean delivery rate on the maternal and/or neonatal outcomes further from now cannot be predicted. Therefore, in the future, we have to pay attention be the changes in Cesarean delivery rate and perinatal outcomes at our institute in Japan.

In conclusion, the Cesarean delivery rate at our institute increased between 2002 and 2012. The incident of low $\mathrm{UApH}$ seemed to be improved associated with the increased Cesarean delivery rate.

\section{Conflict of Interests}

The authors report no conflict of interests. The authors alone are responsible for the content and writing the paper.

\section{References}

[1] E. L. Barber, L. S. Lundsberg, K. Belanger, C. M. Pettker, E. F. Funai, and J. L. Illuzzi, "Indications contributing to the increasing cesarean delivery rate," Obstetrics and Gynecology, vol. 118, no. 1, pp. 29-38, 2011.

[2] F. Menacker and B. E. Hamilton, "Recent trends in cesarean delivery in the United States," NCHS Data Brief, no. 35, pp. 1$8,2010$.

[3] A. Boyle and U. M. Reddy, "Epidemiology of cesarean delivery: the scope of the problem," Seminars in Perinatology, vol. 36, pp. 308-314, 2012.

[4] S. K. Srinivas, C. Fager, and S. A. Lorch, "Evaluating riskadjusted cesarean delivery rate as a measure of obstetric quality," Obstetrics and Gynecology, vol. 115, no. 5, pp. 1007-1013, 2010.

[5] American College of Obstetricians and Gynecologists, "ACOG Practice bulletin no. 115: vaginal birth after previous cesarean delivery," Obstetrics and Gynecology, vol. 116, no. 2, pp. 450-463, 2010.

[6] H. C. Lee, Y. Y. El-Sayed, and J. B. Gould, "Delivery mode by race for breech presentation in the US," Journal of Perinatology, vol. 27, no. 3, pp. 147-153, 2007.

[7] Perinatal Committee Report (in Japanese), "Japan Society of Obstetrics and Gynecology," http://www.jsog.or.jp/.

[8] H. Takahashi, N. Watanabe, R. Sugibayashi et al., "Increased rate of cesarean section in primiparous women aged 40 years or more: a single-center study in Japan," Archives of Gynecology and Obstetrics, vol. 285, no. 4, pp. 937-941, 2012.

[9] S. Suzuki, "Trends in mode of delivery of breech presentation over a 5-year period," Journal of Perinatology, vol. 27, no. 7, article 464, 2007.

[10] American College of Obstetricians and Gynecologists, "ACOG committee opinion No. 265: mode of term singleton breech delivery," Obstetricians and Gynecologists, vol. 98, no. 6, pp. 1189-1190, 2001.

[11] M. Nakata-Konishi, H. Miyake, and S. Suzuki, "Trial of labor after cesarean delivery as an independent risk factor for intrapartum asphyxia in singleton deliveries beyond 37 weeks' gestation," Journal of Nippon Medical School, vol. 79, pp. 362366, 2012. 


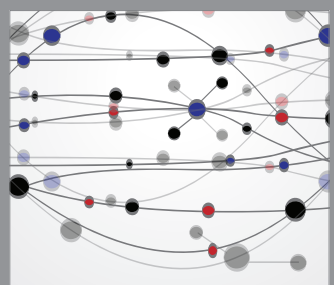

The Scientific World Journal
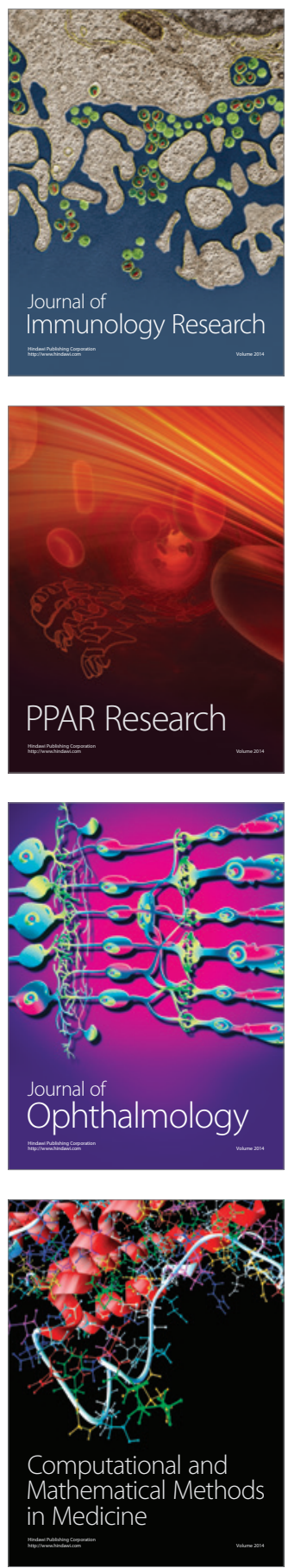

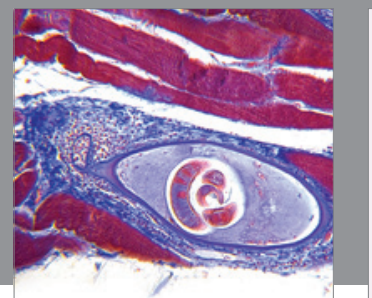

Gastroenterology

Research and Practice
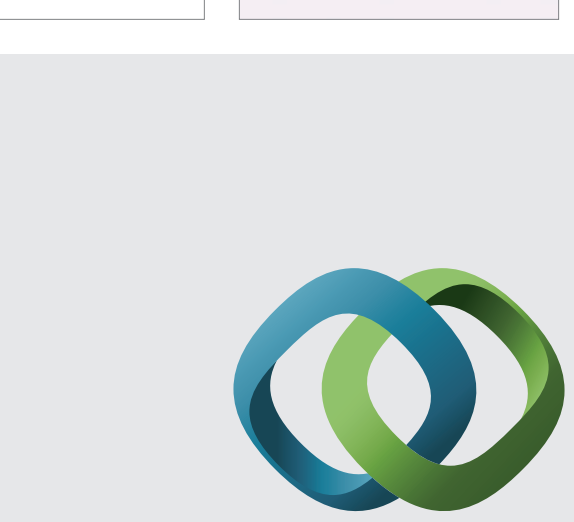

\section{Hindawi}

Submit your manuscripts at

http://www.hindawi.com
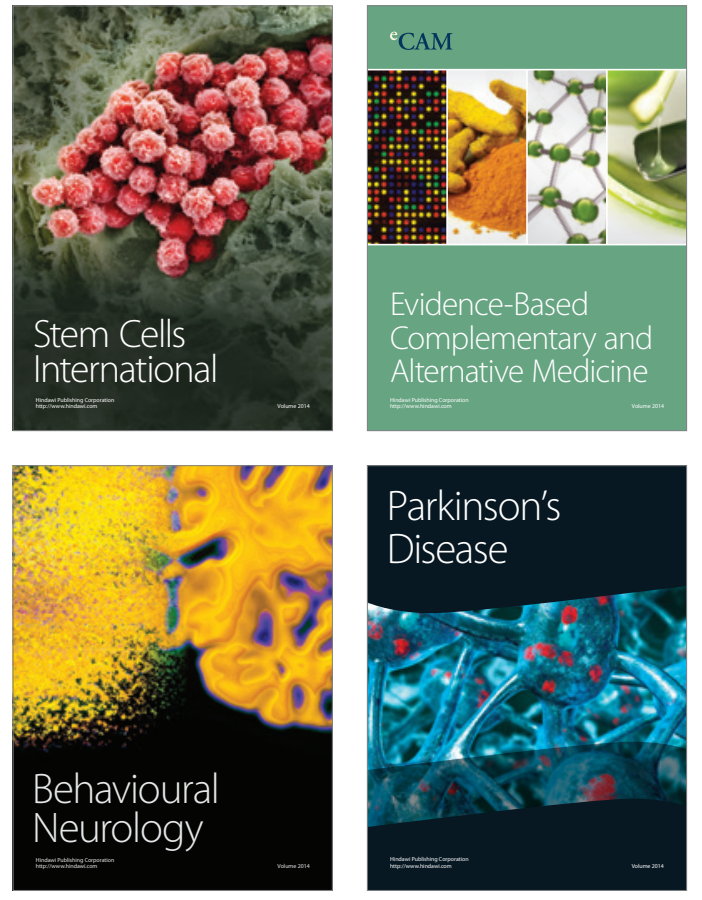
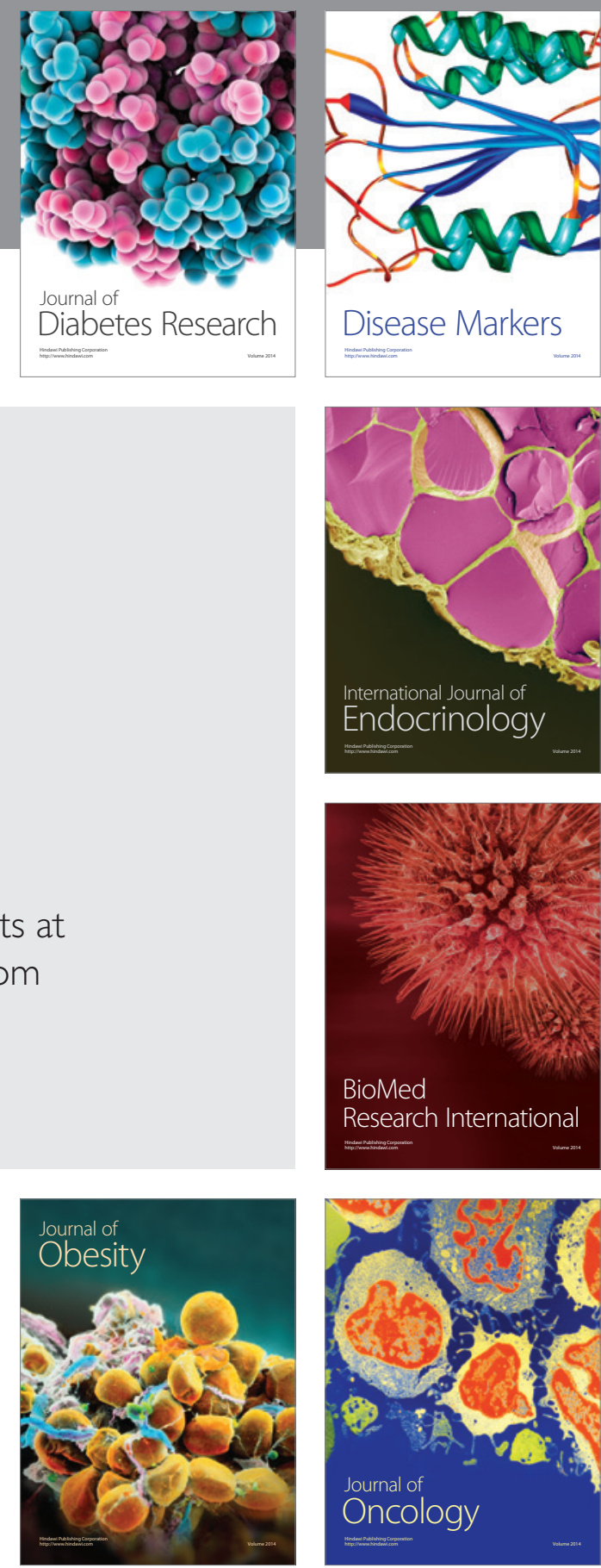

Disease Markers
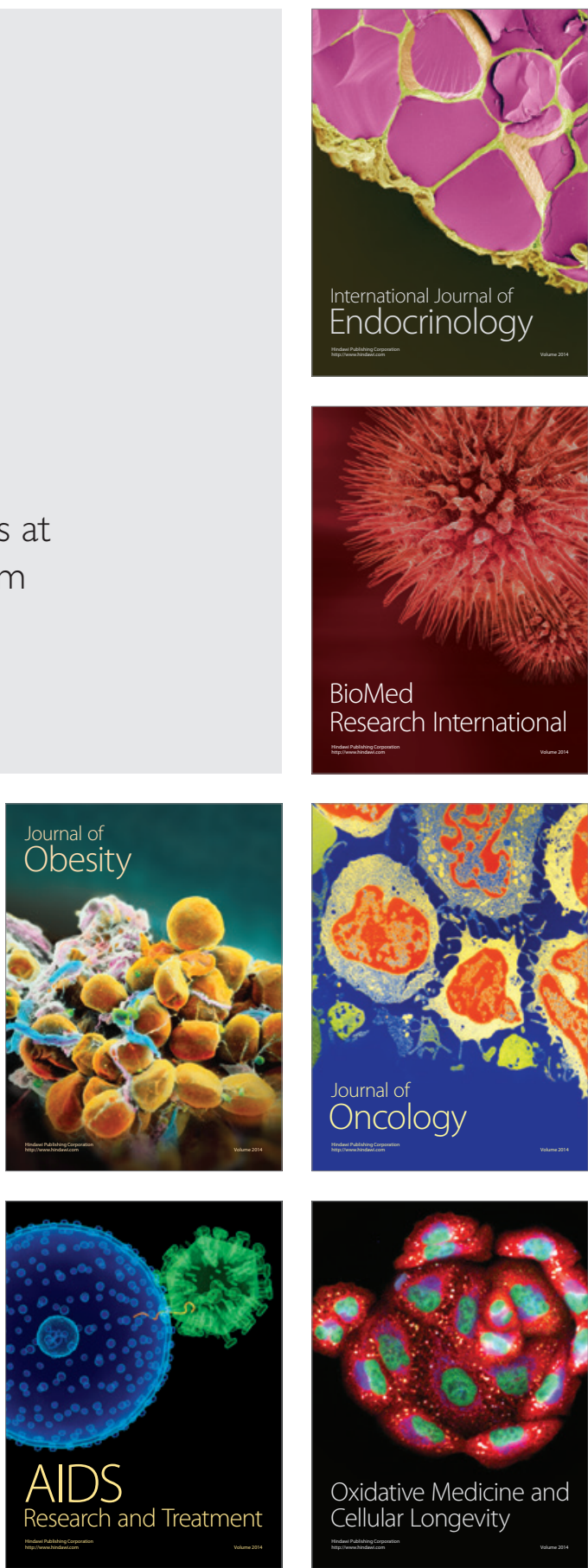